\title{
ANÁLISE DA REPRODUTIBILIDADE DAS CLASSIFICAÇÕES DAS FRATURAS DO PLATÔ TIBIAL
}

\section{ANALYSIS OF THE REPRODUCIBILITY OF TIBIAL PLATEAU FRACTURES' CLASSIFICATION}

Rodrigo Pires e Albuquerque ${ }^{1}$, Vincenzo Giordano ${ }^{2}$, Alexandre Pallottino ${ }^{3}$, Tannous Sassine ${ }^{4}$, Rodrigo Canedo ${ }^{4}$, Juliana Pina ${ }^{4}$, Ney Pecegueiro do Amaral ${ }^{5}$

\section{RESUMO}

Objetivo: Avaliar a concordância interobservador de três classificações utilizadas para a fratura do platô tibial: Schatzker, Grupo AO e Hohl. Método: Nove observadores, subdivididos de acordo com o grau de titulação (seis médicos residentes - MR - e três médicos especialistas - ME), classificaram 50 fraturas do platô tibial. A concordância interobservador foi avaliada pelo coeficiente Kappa. Resultados: Comparando-se os médicos residentes (MR) com os médicos especialistas (ME), observou-se que as três classificações apresentaram concordância de grau fraco e moderado. Entre os MR, as classificações do Grupo AO e de Hohl mostraram relação direta entre aumento no grau de concordância e ano de residência médica, o que foi observado de forma parcial para a classificação de Schatzker. Para a classificação de Hohl, o Kappa variou de 0,344 a 0,577 ; para a classificação do Grupo AO, variou de 0,36 a 0,499; e para a classificação de Schatzker, variou de 0,278 a 0,465. Conclusão: Existe concordância significativa, ao nível de 1\%, entre as três classificações para todos os pares de observadores. Comparando-se os MR com os ME, as três classificações estudadas apresentam concordância de grau fraco e moderado. A classificação de Hohl apresenta a maior concordância entre os MR.

Descritores - Fraturas da tíbia/classificação; Estudos da validação; Avaliação

\section{ABSTRACT}

Objective: To assess the inter-observer agreement of three tibial plateau fracture classifications: Schatzker, AO Group, and Hohl. Methods: Nine physicians of different levels of expertise (six Orthopaedic Residents - OR - and three Orthopaedic Attendings - OA) classified 50 tibial plateau fractures. Results: There was a low to moderate agreement between OR and OA on the three classification systems. A straight correlation was found between the year of Residence and an increase on the level of agreement on the AO group and Hohl classifications. This was partially seen on the Schatzker classification. Kappa ranged from 0.344 to 0.577, 0.36 to 0.499, and 0.278 to 0.465 for the Hohl, AO group, and Schatzker systems, respectively. Conclusion: With a level of 0.1, there was a significant agreement on the three classifications among the physicians. When comparing ORs to OAs, the three studied classifications show low to moderate consistency, with the Hohl classification showing the highest level of agreement between $O R$.

Keywords - Tibial fractures/classification; Validation studies; Evaluation

\section{INTRODUÇÃO}

As fraturas do platô tibial representam 1 a $2 \%$ de todas as fraturas e aproximadamente $8 \%$ das fraturas em idosos ${ }^{(1)}$. Por se tratar de fraturas que envolvem a cartilagem articular, necessitam de redução anatômica e mobilidade precoce, que são preferencialmente obtidas por meio cirúrgico ${ }^{(2)}$. Nesse sentido, a utilização de um sistema de classificação abrangente torna mais compreensível a definição da tática e do acesso cirúrgicos.

De forma conceitual, a proposta da adoção de um sistema de classificação passa por sua capacidade de reprodutibilidade, simplicidade e facilidade de memorização, aju-

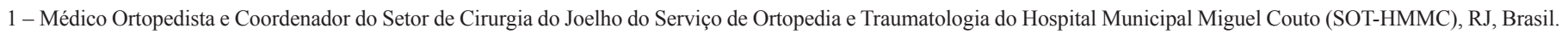

2 - Médico Ortopedista e Coordenador do Programa de Residência Médica do SOT-HMMC/RJ, Brasil.

3 - Médico Ortopedista Assistente do SOT-HMMC/RJ, Brasil.

4-Médico Ortopedista Colaborador do SOT-HMMC/RJ, Brasil.

5 - Chefe do SOT-HMMC/RJ, Brasil.

Trabalho realizado no Serviço de Ortopedia e Traumatologia Professor Nova Monteiro - Hospital Municipal Miguel Couto (SOT-HMMC), Rio de Janeiro, RJ, Brasil. Correspondência: Av. Henrique Dodsworth, 83, apto. 105, CEP: 22061-030, Rio de Janeiro, RJ, Brasil. Tel./fax: (21) 2287-2063. E-mail: rodalbuquerque@ibest.com.br 
dando na escolha do tratamento e na predição prognóstica da lesão existente. Na fratura do platô tibial, as classificações mais utilizadas são a de Schatzker ${ }^{(3)}$, a do Grupo AO (Arbeitsgemeinschaft für Osteosynthesefragen) ${ }^{(4)}$ e a de Hohl ${ }^{(5)}$.

O objetivo desta pesquisa foi avaliar a concordância interobservador nas classificações de Schatzker, do Grupo AO e de Hohl em um grupo de médicos divididos em diferentes graus de experiência profissional.

\section{MÉTODO}

Entre os meses de outubro de 2005 e junho de 2006, no Serviço de Ortopedia e Traumatologia Prof. Nova Monteiro, do Hospital Municipal Miguel Couto (HMMC), foi realizado estudo prospectivo da reprodutibilidade das classificações mais empregadas para as fraturas do platô tibial. Foram avaliadas as radiografias do joelho de 50 indivíduos atendidos no HMMC com diagnóstico de fratura do platô tibial. Todos eram esqueleticamente maduros, com média de idade de 47,9 anos, variando de 21 a 89 anos, sendo 15 mulheres e 35 homens. Vinte e uma fraturas ocorreram no lado direito e 29 , no esquerdo.

Radiografias do joelho do lado da lesão foram obtidas de todos os pacientes, em projeções anteroposterior e lateral, usando protocolo-padrão. Os pacientes foram colocados em posição supina, com o joelho em extensão máxima e a perna em posição neutra; a ampola de raios $\mathrm{X}$ foi colocada a $1 \mathrm{~m}$ de distância do local lesionado com o raio direcionado ortogonalmente ao polo inferior da patela em angulação cefálica de $5^{\circ}$. Na radiografia lateral, o paciente foi mantido com $20^{\circ}$ de flexão do joelho com a distância tubo-filme padronizada em $1 \mathrm{~m}$ e angulação cefálica de $5^{\circ}$. Utilizou-se aparelho de raios X Super $100^{\circledR}$ (Philips, Brasil), com técnica de $50 \mathrm{kV}$ e $31 \mathrm{~mA}$. O exame foi avaliado pelos pesquisadores quanto à qualidade da imagem e repetido caso fosse julgado de má qualidade técnica.

Os exames foram mostrados para um grupo de nove médicos com diferentes graus de experiência em ortopedia e traumatologia. O grupo foi composto de três médicos membros da Sociedade Brasileira de Trauma Ortopédico, considerados como médicos especialistas (ME), e seis médicos residentes (MR), dois do terceiro ano (R3), dois do segundo ano (R2) e dois do primeiro ano (R1). Todos os participantes foram esclarecidos acerca do objetivo desta investigação. O projeto foi enviado à aprovação da Comissão de Ética em Pesquisa da Secretaria Municipal de Saúde do Rio de Janeiro, de acordo com a Resolução 196/96 do Conselho Nacional de Saúde (Diretrizes e Normas Regulamentadoras de Pesquisa Envolvendo Seres Humanos $)^{(6)}$. O estudo foi informado aos pacientes e solicitado um termo de consentimento, que era assinado pelo próprio paciente ou familiar próximo.
A fim de minimizar o viés devido à dificuldade de interpretação ou algum possível esquecimento, as classificações encontravam-se descritas na folha de resposta associadas com desenhos esquemáticos das respectivas classificações, entregue a cada observador, no ato da avaliação das radiografias (Figuras 1, 2 e 3). Não houve limite de tempo para que as radiografias fossem classificadas.

A coleta da análise radiográfica foi realizada de forma cega por um dos autores, que recolheu os formulários e digitalizou as respostas dos nove participantes. A análise estatística foi realizada pela estatística Kappa, que avalia a concordância interobservadores de dados de natureza qualitativa. A hipótese testada é se a concordância $(\rho)$ é igual a zero, isto é, não existe concordância interobservadores. Sabe-se que a estatística Kappa varia entre um valor negativo e 1,0 (Kappa igual a 1 expressa concordância perfeita, Kappa próximo de zero expressa discordância, ou seja, a concordância observada não é melhor do que o acaso). O nível de significância adotado foi $\alpha=1 \%$ (Figuras $1,2,3$ ).

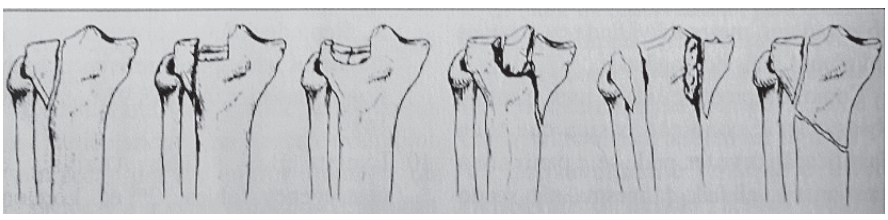

Figura 1 - Classificação de Schatzker

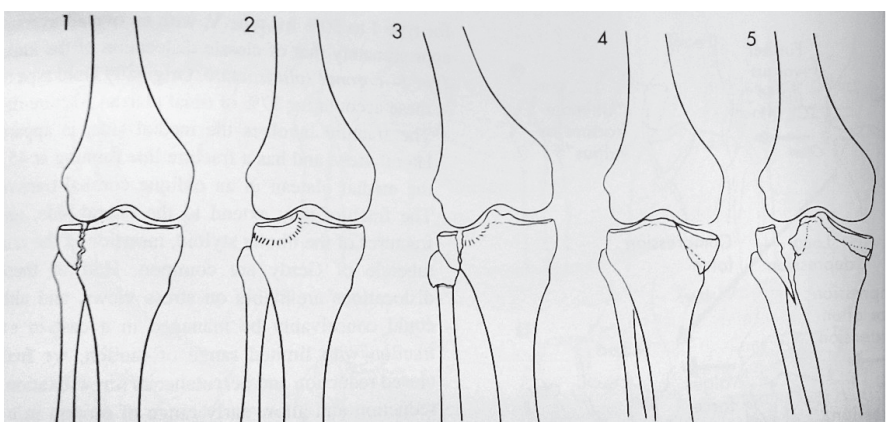

Figura 2 - Classificação de Hohl

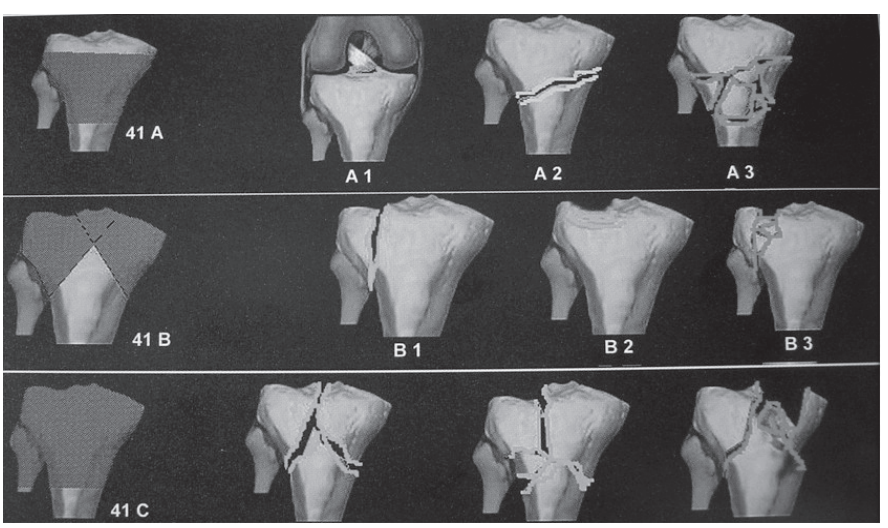

Figura 3 - Classificação AO/ASIF 


\section{RESULTADOS}

As tabelas 1, 2, 3 e 4 fornecem a freqüência (n) e o percentual (\%) das classificações de Schatzker, AO e Hohl dos nove observadores para os 50 pacientes estudados.

Tabela 1 - Análise descritiva da classificação de Schatzker.

\begin{tabular}{l|c|c|c|c|c|c|c|c|c|c|c|c|c|c}
\hline Classificação & \multicolumn{2}{|c|}{ ME } & \multicolumn{2}{|c|}{ R3_1 } & \multicolumn{2}{|c|}{ R3_2 } & \multicolumn{2}{|c|}{ R2_1 } & \multicolumn{2}{|c|}{ R2_2 } & \multicolumn{2}{|c|}{ R1_1 } & \multicolumn{2}{|c}{ R1_2 } \\
\hline & $\mathbf{n}$ & $\%$ & $\mathbf{n}$ & $\%$ & $\mathbf{n}$ & $\%$ & $\mathbf{n}$ & $\%$ & $\mathbf{n}$ & $\%$ & $\mathbf{n}$ & $\%$ & $\mathbf{n}$ & $\%$ \\
\hline Schatzker 1 & 7 & 14 & 10 & 20 & 6 & 12 & 11 & 22 & 10 & 20 & 14 & 28 & 11 & 22 \\
\hline Schatzker 2 & 21 & 42 & 16 & 32 & 10 & 20 & 15 & 30 & 21 & 42 & 17 & 34 & 17 & 34 \\
\hline Schatzker 3 & 7 & 14 & 7 & 14 & 18 & 36 & 6 & 12 & 3 & 6 & 6 & 12 & 4 & 8 \\
\hline Schatzker 4 & 2 & 4 & 5 & 10 & 3 & 6 & 7 & 14 & 4 & 8 & 3 & 6 & 6 & 12 \\
\hline Schatzker 5 & 8 & 16 & 4 & 8 & 4 & 8 & 5 & 10 & 2 & 4 & 3 & 6 & 5 & 10 \\
\hline Schatzker 6 & 5 & 10 & 8 & 16 & 9 & 18 & 6 & 12 & 10 & 20 & 7 & 14 & 7 & 14 \\
\hline
\end{tabular}

ME: médicos especialistas; R3: residente do 3ㅇan; R2: residente do 2oano; R1:residente do 1ㅇ ano

Fonte: SOT-HMMC, 2006.

Tabela 2 - Análise descritiva da classificação do Grupo AO.

\begin{tabular}{l|c|c|c|c|c|c|c|c|c|c|c|c|c|c}
\hline Classificação & \multicolumn{2}{|c|}{ ME } & \multicolumn{2}{|c|}{ R3_1 } & \multicolumn{2}{|c|}{ R3_2 } & \multicolumn{2}{|c|}{ R2_1 } & \multicolumn{2}{|c|}{ R2_2 } & \multicolumn{2}{|c|}{ R1_1 } & \multicolumn{2}{c}{ R1_2 } \\
\hline & $\mathbf{n}$ & $\%$ & $\mathbf{n}$ & $\%$ & $\mathbf{n}$ & $\%$ & $\mathbf{n}$ & $\%$ & $\mathbf{n}$ & $\%$ & $\mathbf{n}$ & $\%$ & $\mathbf{n}$ & $\%$ \\
\hline AO A1 & 0 & 0 & 0 & 0 & 1 & 2 & 0 & 0 & 1 & 2 & 1 & 2 & 1 & 2 \\
\hline AO A2 & 0 & 0 & 1 & 2 & 1 & 2 & 0 & 0 & 1 & 2 & 0 & 0 & 2 & 4 \\
\hline AO B1 & 9 & 18 & 10 & 20 & 6 & 12 & 14 & 28 & 14 & 28 & 14 & 28 & 12 & 24 \\
\hline AO B2 & 8 & 16 & 10 & 20 & 20 & 40 & 9 & 18 & 8 & 16 & 7 & 14 & 10 & 20 \\
\hline AO B3 & 21 & 42 & 17 & 34 & 12 & 24 & 16 & 32 & 16 & 32 & 18 & 36 & 14 & 28 \\
\hline AO C1 & 7 & 14 & 8 & 16 & 5 & 10 & 4 & 8 & 4 & 8 & 6 & 12 & 5 & 10 \\
\hline AO C2 & 2 & 4 & 1 & 2 & 2 & 4 & 4 & 8 & 2 & 4 & 2 & 4 & 0 & 0 \\
\hline AO C3 & 3 & 6 & 3 & 6 & 3 & 6 & 3 & 6 & 4 & 8 & 2 & 4 & 6 & 12 \\
\hline
\end{tabular}

ME: médicos especialistas; R3: residente do 3 o ano; R2: residente do 2 o ano; R1:residente do 1ㅇ ano

Fonte: SOT-HMMC, 2006.

Tabela 3 - Análise descritiva da classificação do Grupo AO agrupada.

\begin{tabular}{|c|c|c|c|c|c|c|c|c|c|c|c|c|c|c|}
\hline \multirow[t]{2}{*}{ Classificação } & \multicolumn{2}{|c|}{ ME } & \multicolumn{2}{|c|}{ R3_1 } & \multicolumn{2}{|c|}{ R3_2 } & \multicolumn{2}{|c|}{ R2_1 } & \multicolumn{2}{|c|}{ R2_2 } & \multicolumn{2}{|c|}{ R1_1 } & \multicolumn{2}{|c|}{ R1_2 } \\
\hline & $\mathrm{n}$ & $\%$ & $n$ & $\%$ & $\mathrm{n}$ & $\%$ & $\mathrm{n}$ & $\%$ & $\mathrm{n}$ & $\%$ & $n$ & $\%$ & $n$ & $\%$ \\
\hline $\mathrm{AO} A 1 / \mathrm{A} 2$ & 0 & 0 & 1 & 2 & 2 & 4 & 0 & 0 & 2 & 4 & 1 & 2 & 3 & 6 \\
\hline $\mathrm{AO} B 1$ & 9 & 18 & 10 & 20 & 6 & 12 & 14 & 28 & 14 & 28 & 14 & 28 & 12 & 24 \\
\hline $\mathrm{AO} B 2$ & 8 & 16 & 10 & 20 & 20 & 40 & 9 & 18 & 8 & 16 & 7 & 14 & 10 & 20 \\
\hline $\mathrm{AOB} 3$ & 21 & 42 & 17 & 34 & 12 & 24 & 16 & 32 & 16 & 32 & 18 & 36 & 14 & 28 \\
\hline $\mathrm{AOC1}$ & 7 & 14 & 8 & 16 & 5 & 10 & 4 & 8 & 4 & 8 & 6 & 12 & 5 & 10 \\
\hline $\mathrm{AO}$ C2/C3 & 5 & 10 & 4 & 8 & 5 & 10 & 7 & 14 & 6 & 12 & 4 & 8 & 6 & 12 \\
\hline
\end{tabular}

Tabela 4 - Análise descritiva da classificação de Hohl.

\begin{tabular}{|c|c|c|c|c|c|c|c|c|c|c|c|c|c|c|}
\hline \multirow[t]{2}{*}{ Classificação } & \multicolumn{2}{|c|}{ ME } & \multicolumn{2}{|c|}{ R3_1 } & \multicolumn{2}{|c|}{ R3_2 } & \multicolumn{2}{|c|}{ R2_1 } & \multicolumn{2}{|c|}{ R2_2 } & \multicolumn{2}{|c|}{ R1_1 } & \multicolumn{2}{|c|}{ R1_2 } \\
\hline & $\mathrm{n}$ & $\%$ & $n$ & $\%$ & $\mathrm{n}$ & $\%$ & $\mathrm{n}$ & $\%$ & $n$ & $\%$ & $\mathrm{n}$ & $\%$ & $n$ & $\%$ \\
\hline Hohl 1 & 7 & 14 & 10 & 20 & 6 & 12 & 11 & 22 & 9 & 18 & 15 & 30 & 11 & 22 \\
\hline Hohl 2 & 9 & 18 & 6 & 12 & 18 & 36 & 6 & 12 & 5 & 10 & 5 & 10 & 1 & 2 \\
\hline Hohl 3 & 20 & 40 & 16 & 32 & 12 & 24 & 16 & 32 & 22 & 44 & 20 & 40 & 21 & 42 \\
\hline Hohl 4 & 2 & 4 & 5 & 10 & 1 & 2 & 7 & 14 & 4 & 8 & 1 & 2 & 4 & 8 \\
\hline Hohl 5 & 12 & 24 & 13 & 26 & 13 & 26 & 10 & 20 & 10 & 20 & 9 & 18 & 13 & 26 \\
\hline
\end{tabular}

As tabelas 5, 6 e 7 fornecem a concordância observada (em \%), estatística Kappa, erro padrão de Kappa (EP) e o nível descritivo ( $p$-valor) para cada par de observadores considerado para as classificações de Schatzker, AO e Hohl, respectivamente.

A concordância observada corresponde ao percentual (\%) de respostas idênticas no total de 50 pacientes avaliados. Por exemplo, no total de 50 pacientes, segundo a classificação de Schatzker, o R3_1 concordou em 58\% dos casos com os ME.

Tabela 5 - Análise interobservador para classificação de Schatzker.

\begin{tabular}{|c|c|c|c|c|}
\hline Obs 1 x Obs 2 & $\begin{array}{c}\text { Concordância } \\
\text { observada }\end{array}$ & $\begin{array}{l}\text { Coeficiente } \\
\text { Kappa }\end{array}$ & EP de Kappa & $p$-valor \\
\hline ME x R3_1 & 58,0 & 0,465 & 0,067 & $<0,0001$ \\
\hline ME x R3_2 & 52,0 & 0,411 & 0,062 & $<0,0001$ \\
\hline ME x R2_1 & 48,0 & 0,344 & 0,066 & $<0,0001$ \\
\hline ME x R2_2 & 50,0 & 0,340 & 0,068 & $<0,0001$ \\
\hline ME x R1_1 & 44,0 & 0,278 & 0,068 & $<0,0001$ \\
\hline ME x R1_2 & 54,0 & 0,411 & 0,067 & $<0,0001$ \\
\hline R3_1 $x$ R3_2 & 52,0 & 0,415 & 0,063 & $<0,0001$ \\
\hline R3_1 x R2_1 & 54,0 & 0,426 & 0,067 & $<0,0001$ \\
\hline R3_1 $\times$ R2_2 & 64,0 & 0,535 & 0,070 & $<0,0001$ \\
\hline R3_1 x R1_1 & 54,0 & 0,414 & 0,070 & $<0,0001$ \\
\hline R3_1 x R1_2 & 56,0 & 0,446 & 0,068 & $<0,0001$ \\
\hline R3_2 x R2_1 & 54,0 & 0,447 & 0,060 & $<0,0001$ \\
\hline R3_2 x R2_2 & 52,0 & 0,419 & 0,059 & $<0,0001$ \\
\hline R3_2 x R1_1 & 54,0 & 0,440 & 0,062 & $<0,0001$ \\
\hline R3_2 x R1_2 & 40,0 & 0,283 & 0,059 & $<0,0001$ \\
\hline $\mathrm{R} 2 \_1 \times \mathrm{R} 2 \_2$ & 54,0 & 0,413 & 0,068 & $<0,0001$ \\
\hline R2_1 x R1_1 & 50,0 & 0,368 & 0,068 & $<0,0001$ \\
\hline $\mathrm{R} 2 \_1 \times \mathrm{R} 1 \_2$ & 44,0 & 0,297 & 0,068 & $<0,0001$ \\
\hline $\mathrm{R} 2 \_2 \times \mathrm{R} 1 \_1$ & 60,0 & 0,473 & 0,073 & $<0,0001$ \\
\hline R2_2 x R1_2 & 62,0 & 0,504 & 0,071 & $<0,0001$ \\
\hline R1_1 x R1_2 & 66,0 & 0,564 & 0,070 & $<0,0001$ \\
\hline
\end{tabular}

Obs:observador; ME: médicos especialistas; R3: residente do 3 o ano; R2: residente do $2^{\circ}$ ano; R1:residente do 1ำ ano EP: Erro Padrão.

Fonte: SOT-HMMC, 2006. 
Tabela 6 - Análise interobservador para classificação do Grupo AO.

\begin{tabular}{l|c|c|c|c}
\hline Obs 1 x Obs 2 & $\begin{array}{c}\text { Concordância } \\
\text { observada }\end{array}$ & $\begin{array}{c}\text { Coeficiente } \\
\text { Kappa }\end{array}$ & EP de Kappa & $p$-valor \\
\hline MEx R3_1 & 62,0 & 0,499 & 0,074 & $<0,0001$ \\
\hline MExR3_2 & 58,0 & 0,468 & 0,067 & $<0,0001$ \\
\hline MExR2_1 & 62,0 & 0,501 & 0,074 & $<0,0001$ \\
\hline MExR2_2 & 52,0 & 0,374 & 0,072 & $<0,0001$ \\
\hline MExR1_1 & 54,0 & 0,388 & 0,075 & $<0,0001$ \\
\hline MExR1_2 & 50,0 & 0,360 & 0,070 & $<0,0001$ \\
\hline R3_1 xR3_2 & 64,0 & 0,544 & 0,068 & $<0,0001$ \\
\hline R3_1 xR2_1 & 60,0 & 0,484 & 0,072 & $<0,0001$ \\
\hline R3_1 x R2_2 & 56,0 & 0,436 & 0,071 & $<0,0001$ \\
\hline R3_1 xR1_1 & 64,0 & 0,531 & 0,073 & $<0,0001$ \\
\hline R3_1 x R1_2 & 54,0 & 0,418 & 0,070 & $<0,0001$ \\
\hline R3_2 x R2_1 & 56,0 & 0,447 & 0,067 & $<0,0001$ \\
\hline R3_2 x R2_2 & 54,0 & 0,428 & 0,065 & $<0,0001$ \\
\hline R3_2 x R1_1 & 54,0 & 0,427 & 0,065 & $<0,0001$ \\
\hline R3_2 x R1_2 & 40,0 & 0,250 & 0,067 & $<0,0001$ \\
\hline R2_1 x R2_2 & 56,0 & 0,426 & 0,074 & $<0,0001$ \\
\hline R2_1 xR1_1 & 52,0 & 0,369 & 0,075 & $<0,0001$ \\
\hline R2_1 xR1_2 & 48,0 & 0,335 & 0,072 & $<0,0001$ \\
\hline R2_2 x R1_1 & 58,0 & 0,450 & 0,074 & $<0,0001$ \\
\hline R2_2 xR1_2 & 56,0 & 0,440 & 0,071 & $<0,0001$ \\
\hline R1_1 xR1_2 & 66,0 & 0,565 & 0,071 & $<0,0001$ \\
\hline
\end{tabular}

Obs:observador; ME: médicos especialistas; R3: residente do 3 o ano; R2: residente do 2 o ano; R1: residente do $1^{\circ}$ ano EP: erro padrão.

Fonte: SOT-HMMC, 2006.

A classificação do Grupo AO foi analisada de forma agrupada (Tabela 3), devido ao número muito pequeno de casos observados nas categorias A1 e A2, e entre os $\mathrm{C} 1, \mathrm{C} 2$ e $\mathrm{C} 3$.

Quando se compararam os MR com os ME, observouse que as três classificações apresentaram concordância fraca a moderada; nenhuma com boa qualidade.

As classificações do Grupo AO e de Hohl mostraram aumento no grau de concordância à medida que aumentam os anos de residência médica. Resultado observado de forma parcial para a classificação de Schatzker.

Com base no comentário anterior, a classificação de Hohl atingiu melhores resultados (Kappa variando de 0,344 a 0,577) do que a classificação do Grupo AO (Kappa variando de 0,36 a 0,499), e a de Schatzker (Kappa variando de 0,278 a 0,465$)$.
Tabela 7 - Análise interobservador para classificação de Hohl.

\begin{tabular}{|c|c|c|c|c|}
\hline Obs 1 x Obs 2 & $\begin{array}{l}\text { Concordância } \\
\text { observada }\end{array}$ & $\begin{array}{l}\text { Coeficiente } \\
\text { Kappa }\end{array}$ & EP de Kappa & $p$-valor \\
\hline ME x R3_1 & 68,0 & 0,577 & 0,076 & $<0,0001$ \\
\hline ME x R3_2 & 64,0 & 0,526 & 0,076 & $<0,0001$ \\
\hline ME x R2_1 & 60,0 & 0,478 & 0,073 & $<0,0001$ \\
\hline ME x R2_2 & 60,0 & 0,452 & 0,078 & $<0,0001$ \\
\hline ME x R1_1 & 52,0 & 0,348 & 0,078 & $<0,0001$ \\
\hline ME x R1_2 & 52,0 & 0,344 & 0,077 & $<0,0001$ \\
\hline R3_1 x R3_2 & 60,0 & 0,491 & 0,070 & $<0,0001$ \\
\hline R3_1 $x$ R2_1 & 62,0 & 0,509 & 0,074 & $<0,0001$ \\
\hline R3_1 $x$ R2_2 & 66,0 & 0,547 & 0,076 & $<0,0001$ \\
\hline R3_1 x R1_1 & 58,0 & 0,441 & 0,076 & $<0,0001$ \\
\hline R3_1 x R1_2 & 60,0 & 0,462 & 0,078 & $<0,0001$ \\
\hline R3_2 x R2_1 & 54,0 & 0,424 & 0,067 & $<0,0001$ \\
\hline R3_2 $x$ R2_2 & 54,0 & 0,413 & 0,069 & $<0,0001$ \\
\hline R3_2 x R1_1 & 54,0 & 0,414 & 0,069 & $<0,0001$ \\
\hline R3_2 x R1_2 & 42,0 & 0,272 & 0,064 & $<0,0001$ \\
\hline $\mathrm{R} 2 \_1 \times \mathrm{R} 2 \_2$ & 54,0 & 0,392 & 0,075 & $<0,0001$ \\
\hline R2_1 x R1_1 & 50,0 & 0,338 & 0,075 & $<0,0001$ \\
\hline $\mathrm{R} 2 \_1 \times \mathrm{R} 1 \_2$ & 52,0 & 0,361 & 0,076 & $<0,0001$ \\
\hline R2_2 x R1_1 & 60,0 & 0,446 & 0,080 & $<0,0001$ \\
\hline R2_2 x R1_2 & 60,0 & 0,441 & 0,081 & $<0,0001$ \\
\hline $\mathrm{R} 1 \_1 \times \mathrm{R} 1 \_2$ & 66,0 & 0,525 & 0,082 & $<0,0001$ \\
\hline
\end{tabular}

Obs:observador; ME: médicos especialistas; R3: residente do $3^{\circ}$ ano; R2: residente do 2o ano; R1:residente do 10 ano EP: erro padrão.

Fonte: SOT-HMMC, 2006.

\section{DISCUSSÃO}

As classificações em ortopedia e traumatologia são ferramentas que auxiliam a uniformizar uma linguagem internacional na abordagem das lesões. O que geralmente acontece é que, estabelecida uma classificação para determinada lesão, nem sempre baseada em casuística relevante e representativa, alguns casos não se enquadram nos tipos descritos ou classificados. Weber, em seu clássico estudo sobre fraturas maleolares, reservou um subgrupo para as lesões “inclassificáveis”, aquelas que não poderiam ser encaixadas em classes ou grupos, devido a sua peculiaridade ${ }^{(7)}$. Algumas classificações ao longo do tempo foram substituídas por outras mais completas.

No Brasil, ainda hoje existem poucos serviços que dispõem de tomografia computadorizada ou ressonância magnética de rotina; daí a necessidade de um estudo 
avaliando as classificações radiográficas nas fraturas do platô tibial. Dirschl e Patrick ${ }^{(8)}$ observaram que as classificações para as fraturas do platô tibial apresentam baixa reprodutibilidade interobservador, dispertando o interesse de nossa pesquisa.

Walton et $a l^{(9)}$ avaliaram 53 radiografias do joelho em pacientes com fraturas do platô tibial e compararam a classificação de Schatzker com a do Grupo AO. Concluíram que a classificação AO foi mais reprodutível que a classificação de Schatzker na análise interobservador, corroborando nosso estudo; observaram que ambas as classificações foram originalmente baseadas em estudos radiográficos, demonstrando a importância de nossa pesquisa. Charalambous et al $l^{(10)}$ observaram que as classificações de Schatzker e AO foram pouco reprodutíveis na análise intra e interobservador, confirmando o resultado de nossa pesquisa.

Mandarino et $a l^{(11)}$ avaliaram 20 radiografias do joelho em pacientes com fraturas do platô tibial num estudo retrospectivo e analisaram a reprodutibilidade da classificação de Schatzker. Concluíram que a classificação de Schatzker é moderadamente reprodutível na análise interobservador, mesmo após agrupá-los em grupos mais homogêneos, comprovando o que evidenciamos em nosso estudo.

Martin et $a l^{(12)}$ analisaram a reprodutibilidade interobservador da classificação AO em 56 radiografias de fraturas do platô tibial. Concluíram que a classificação AO na fraturas do platô tibial é reprodutível e que a concordância da avaliação radiográfica convencional é dependente da experiência do observador. Quando correlacionamos com nosso estudo, observamos que, à medida que au- mentávamos o grau de experiência dos médicos, melhorávamos a concordância, revalidando a nossa pesquisa.

Khan et $a l^{(13)}$ elaboraram uma nova classificação para avaliar fraturas do platô tibial. Foram avaliados 80 pacientes com fraturas do platô tibial de forma retrospectiva. Observaram que as classificações já existentes apresentavam falhas e, às vezes, graduavam diferentes fraturas num mesmo subgrupo. Quando correlacionamos com nosso trabalho, observamos a dificuldade de graduar as fraturas com fragmento posterior do platô tibial nas classificações existentes.

Rafii et $a l^{(14)}$ demonstraram superioridade da tomografia computadorizada (TC) sobre a radiologia convencional nas fraturas do platô tibial. A TC, segundo os autores, é um método confiável para avaliação e classificação das fraturas do platô tibial. Apesar disso, é realidade na maioria dos hospitais que a TC não é disponível, além de ser exame de alto custo e grande exposição à radiação.

Maripuri et $a l^{(15)}$ realizaram um estudo similar ao nosso, onde compararam as classificações de Schatzker, AO e Hohl. Observaram que nenhuma dessas classificações é ideal. Quando analisamos nosso estudo, verificamos que há concordância fraca a moderada.

\section{CONCLUSÕES}

Existe concordância significativa, ao nível de 1\%, entre as três classificações para todos os pares de observadores. Comparando-se os MR com os ME, as três classificações estudadas apresentam concordância de grau fraco e moderado. A classificação de Hohl apresenta a maior concordância entre os MR.

\section{REFERÊNCIAS}

1. Jacofsky DJ, Haidukerwych GJ. Tibia plateau fractures. In: Scott WN. Insall \& Scott surgery of the knee. Philadelphia: Churchill Livingstone; 2006. p.1133-46.

2. Stover MD, Kellam JF. Fraturas articulares: princípios. In: Ruëdi TP, Murphy WM. Princípios AO do tratamento de fraturas. Porto Alegre: Artmed; 2002. p.105-19.

3. Schatzker J, Mc Broom R, Bruce D. The tibial plateau fracture: the Toronto experience 1968-1975. Clin Orthop Relat Res. 1979;(138):94-104.

4. Murphy WM, Leu D. Fraturas articulares: princípios. In: Ruëdi TP, Murphy WM. Princípios AO do tratamento de fraturas. Porto Alegre: Artmed; 2002. p.45-58

5. Hohl M, Luck J. Fractures of the tibial condyle: a clinical and experimental study. J Bone Joint Surg Am. 1956;38(5):1001-18.

6. Comissão Nacional de Ética em Pesquisa (CONEP). http://conselho.saúde.gov. br/comissão/eticapesq.htm. Acessado em dezembro de 2006.

7. Franco JS, Musafir M. Classificação das fraturas mais freqüente. Rio de Janeiro: Schering- Plough; 1991.

8. Dirschl DR, Patrick DA. Injury severity assessment in tibial plateau fractures. Clin Orthop Relat Res. 2004;(423):85-92.

9. Walton NP, Harish S, Roberts C, Blundell C. AO or Schatzker? How reliable

is classification of tibial plateau fractures? Arch Orthop Trauma Surg. 2003; 123(8):396-8.

10. Charalambous CP, Tryfonidis M, Alvi F, Moran M, Fang C, Samaraji R, Hirst P. Inter- and intra-obsever variation of the Schatzker and AO/OTA classifications of tibial plateau fractures and a proposal of a new classification system. Ann R Coll Surg Engl. 2007;89(4):400-4.

11. Mandarino M, Pessoa A, Guimarães JAM. Avaliação da reprodutibilidade da classificação de Schatzker para as fraturas do planalto tibial. R INTO. 2004; 2(2):11-8.

12. Martin J, Marsh JL, Nepola JV, DirschI DR, Hurwitz S, DeCoster TA. Radiographic fratcure assessments: Which ones can we reliably make? J Orthop Trauma. 2000;14(6):379-85.

13. Khan RMS, Khan SH, Ahmad AJ, Umar M. Tibial Plateau Fractures: A New Classification Scheme. Clin Orthop Relat Res. 2000;(375):231-42.

14. Rafii M, Lamont JG, Firooznia H. Tibial plateau fractures: CT evaluation and classification. Crit Rev Diagn Imaging. 1987;27(2):91-112.

15. Maripuri SN, Rao P, Manoj-Thomas A, Mohanty K. The classifications systems for tibial plateau fractures: How reliable are they? Injury. [serial on the internet]. 2008. Avaliable from ://www.elsevier.com/locate/injury 